\title{
A Rare Coincidence of Turner syndrome and Bronchiectasis: Case Report
}

\author{
Mohammad Almoshantaf ${ }^{1}$, Sarya Swed ${ }^{2}$, Weaam Ezzdean ${ }^{1}$, Fateh Kashkash ${ }^{3}$, Hazem \\ Ghaith $^{4}$, Bisher Sawaf ${ }^{5}$, and Karam Motawea ${ }^{6}$ \\ ${ }^{1}$ Ibn Al-Nafees Hospital \\ ${ }^{2}$ University of Aleppo Faculty of Medicine \\ ${ }^{3}$ University of Aleppo \\ ${ }^{4}$ Al-Azhar University Faculty of Medicine \\ ${ }^{5}$ Syrian Private University \\ ${ }^{6}$ Alexandria University Faculty of Medicine
}

March 5, 2022

\author{
Abstract \\ A Rare Coincidence of Turner syndrome and Bronchiectasis: Case Report \\ A Rare Coincidence of Turner syndrome and Bronchiectasis: Case Report \\ Mohammad Badr Almoshantaf ${ }^{1}$, Sarya Swed ${ }^{2}$, Weaam Ezzdean ${ }^{3}$, Fateh Kashkash ${ }^{4}$, Hazem S. Ghaith ${ }^{5}$, \\ Karam R. Motawea ${ }^{6}$ \\ Department of Neurosurgery, Ibn Al-Nafees Hospital, Damascus, Syria Baderalmoushantaf1995@gmail.com \\ Faculty Of Medicine, Aleppo University, Aleppo, Syria. \\ saryaswed1@gmail.com \\ Department of Urology, Al-Hilal Hospital, Damascus, Syria. \\ Weaamezzdean1@gmail.com \\ Department of pulmonology , Aleppo University Hospital , Aleppo , Syria Fatehaminkashkash@gmail.com \\ Faculty of Medicine, Al-Azhar University, Cairo, Egypt \\ hazemghaith11@gmail.com \\ Faculty of medicine, Alexandria University, Egypt. \\ karammetawea450@gmail.com
}

Corresponding author: Sarya Swed

Faculty Of Medicine, Aleppo University, Aleppo, Syria. [saryaswed1@gmail.com]

\section{Introduction}

Turner syndrome (TS) is a condition seen in phenotypic females, which results when one of the X chromosomes (sex chromosomes) is missing or partially missing and has a prevalence of 1 in 2000 to 2500 live-born 
female children [1]. Clinical presentation varies significantly in Girls with TS especially in those with lower degrees of mosaicism for monosomy $\mathrm{X}$, who may not present with the classic phenotype [2]. Clinical manifestations of TS might include the following common signs and symptoms: short stature, pubertal delay/ovarian insufficiency, cardiac and renal abnormalities, sensorineural hearing loss, ophthalmologic problems, thyroid abnormalities, metabolic syndrome, inflammatory bowel disease, and neurocognitive issues [3]. The diagnosis of TS can be established at a wide range of ages [4,5]. Prenatally, the ultrasound findings of increased nuchal translucency, cystic hygroma, left-sided obstructive cardiac anomalies (especially coarctation of the aorta) in any fetus are highly suggestive of TS and test to confirm the diagnosis prenatally by amniocentesis or chorionic villous sampling is necessary [6]. Webbed neck, lymphedema, or coarctation of the aorta in infancy should prompt a peripheral blood karyotype to rule out TS [6]. However, several girls have delayed diagnosis in late adolescence or early adulthood with some estimates indicating that up to $38 \%$ of TS patients are diagnosed in adulthood [7]. Diagnosis is made by sex chromatin [Barr body] detection and chromosomal karyotyping is necessary for diagnosis and to identify the fraction of ' $\mathrm{Y}$ ' chromosome elements.

The associations of TS with congenital heart disease have been described widely in the literature. Up to one-third of the patients with karyotype 45, X may have coarctation of the aorta: In addition, patients with hypertrophic cardiomyopathy, septal defects, dextrocardia, and anomalous pulmonary venous drainage have been reported anecdotally [8].

Bronchiectasis which is a disease that presents clinically with repeated respiratory infections requiring antibiotics, disabling productive cough, shortness of breath, and hemoptysis, has rarely been reported to be associated with Turner syndrome in the medical literature [8]. The disease primarily involves the bronchi and bronchioles described as a vicious circle of transmural infection and inflammation with mediator release, and it's also related to retained inflammatory secretions and microbes in the small airways that would cause obstruction damaging the airway leading to recurrent infection [9]. However, treatment in clinical practice is still based on antibiotics, pulmonary rehabilitation, and surgery in rare cases, a lot of new treatment has been suggested over the last few years [9].

\section{Case presentation}

A 32-year-old female presented to emergency department with respiratory complaints. She reported repeated chest pain and mild dyspnea. Her medical history involves TS, Hypertension, and chronic kidney disease, which requires dialysis three times per week. Her stature is short and her chest is broad, which is consistent with TS. On physical examination, orthopnea and bilateral fine crackles has been noticed. Room air oxygen saturation was low. COVID-19 has been excluded after doing PCR test. She was admitted in intensive care unit due to unstable overall condition.

Initial blood works showed markedly elevated white blood cells (15.17 109/L) with neutrophils dominance (77.6\%), Hemoglobin (9.6 g/dL), Hematocrit $(28.3 \%)$, CRP (93 mg/L), and LDH (809 U/L). Other tests have been done including: Procalcitonin $(11.5 \mathrm{ng} / \mathrm{ml})$, IL-6 $(62.3 \mathrm{ng} / \mathrm{ml})$, and D-Dimer $(7.31 \mathrm{ng} / \mathrm{ml})$. Urea, Creatinine, and electrolytes are usually mildly elevated before dialysis. Tuberculosis has been excluded by Gene-Xpert.

Chest X-ray revealed bilateral peripheral infiltrations and ground glass opacities[Figure 1]. Infective pneumonia with hypoxia was the initial diagnosis. Also, a high anion gap metabolic acidosis was complicating her case. Later, a CT scan was ordered and revealed clear signs of bilateral bronchiectasis[Figure 2]. An echocardiogram showed slight left ventricular hypertrophy, unsignificant right atrial enlargement, and no coarctation of the aorta.

During her stay in the intensive care unit, she has been treated with broad-spectrum antibiotics, as ceteriaxone and metronidazole, non-invasive oxygen therapy, and repeated dialysis. She had a slow recovery process. Despite that, she has finally showed signs of clinical improvement and was discharged without any need of oxygen supplementation or bronchodilators.

\section{Conclusion}


The coexistence Turner syndrome with bronchiectasis is a rare combination that increase the awareness of clinical doctors especially pulmonologists to follow accurate treatment and suitable diagnosis techniques if they occupied this case in clinic or hospital, in addition to check the medical history perfectly.

\section{Discussion}

This case is a presentation of rare association between TS and bronchiectasis. To the extent of our knowledge, this coexistence is rare and hardly ever reported in the literature.

There are a large number of etiologic factors that have been described to cause bronchiectasis [10]. Rarely enough, a case of ciliary aplasia was reported as a cause for bronchiectasis in TS [11]. Etiology of our case's bronchiectasis has not been determined. Both cystic fibrosis and ciliary aplasia were excluded. Bronchiectasis etiology is possible to be congenital. However, as there is only a little data on this association, TS cannot be established yet as a primary cause for congenital bronchiectasis and further research should be done to study this correlation [12].

The 32-old patient is amenorrheic with underdeveloped female organs. She has never received hormone replacement therapy (HRT). It might be beneficial for future studies to consider following the absence of HRT when dealing with TS and bronchiectasis.

She has never reported similar respiratory symptoms before and medical history is remarkable in terms of repeated upper or lower airway infections. Infective pneumonia was treated by regular protocol without particular considerations toward TS. It is worth mentioning that her recovery process was noticeably slower than non-TS patients with similar presentations. Because of her overall vulnerability due to chronic diseases, her slow recovery cannot be restrictedly addressed to TS. After one year of discharge, no recurrence has been reported to the moment.

\section{Declarations}

*Ethics approval and consent to participate

Ethical approval was given by the Aleppo University Hospital. Consent to participate was taken directly from the patient.

\section{*Consent for publication}

Written informed consent was obtained from the patient for the publication of this case report and any accompanying images. A copy of the written consent is available for review by the Editor of this journal.

\section{*Availability of data and material}

All data generated or analyzed are included in this article

\section{*Competing interests}

No conflict of interest exits in the submission of this manuscript.

\section{*Funding}

Not applicable

\section{*Authors' contributions}

All authors have read and approved the manuscript

MBA: Took the lead in writing and reviewing the manuscript.

SS, WE, KRM , FK , HSG : Helped in writing and reviewing the manuscript.

\section{*Acknowledgements}

Our sincere thanks to all the doctors and nurses who participated in the treatment of this case. 


\section{References:}

1. Nielsen, J, Wohlert, M. Chromosome abnormalities found among 34,910 newborn children: results from a 13-year incidence study in Arhus, Denmark. Hum Genet 1991; DOI: 10.1007/BF01213097

2. Claus H Gravholt 1 2, Niels H Andersen 3, Gerard S Conway 4, Olaf M Dekkers 5, Mitchell E Geffner 6, Karen O Klein 7, Angela E Lin 8, Nelly Mauras 9, Charmian A Quigley 10, Karen Rubin 11, David E Sandberg 12, Theo C J Sas 13 14, Michael Silberbach 15, Viveca Söderström-Anttila 16, Kirstine Stochholm 1 17, Janielle A van Alfen-van derVelden 18, Joachim Woelfle 19, Philippe F Backeljauw 20, International Turner Syndrome Consensus Group; Clinical practice guidelines for the care of girls and women with Turner syndrome: proceedings from the 2016 Cincinnati International Turner Syndrome Meeting; 10.1530/EJE-17-0430

3. Roopa Kanakatti Shankar, Philippe F. Backeljauw: Current best practice in the management of Turner syndrome 2017: doi.org/10.1177/2042018817746291

4. Kirstine Stochholm 1, Svend Juul, Knud Juel, Rune Weis Naeraa, Claus Højbjerg Gravholt; Prevalence, incidence, diagnostic delay, and mortality in Turner syndrome 2006; DOI: 10.1210/jc.2006-0558

5. Jordan E Pinske: Clinical review: Turner syndrome: updating the paradigm of clinical care 2012; DOI: $10.1210 /$ jc. $2012-1245$

6. Carolyn A. Bondy: Care of Girls and Women with Turner Syndrome: A Guideline of the Turner Syndrome Study Group 2007; https://doi.org/10.1210/jc.2006-1374

7. Claus H Gravholt; Clinical practice in Turner syndrome 2005; Ihttps://doi.org/10.1038/ncpendmet0024

8. Patil, Mahadev; Maske, Manoj; Joshi, J. M.; BRONCHIECTASIS IN TURNER'S SYNDROME 2006; 10.4103/0970-2113.44403

9. Prof James D Chalmers Ph.D. Sanjay H Chotirmall Ph.D.; Bronchiectasis: new therapies and new perspectives 2021: https://doi.org/10.1016/S2213-2600(18)30053-

10. King, P. (2011). Pathogenesis of bronchiectasis. Paediatric Respiratory Reviews, 12(2), 104-110. https://doi.org/10.1016/J.PRRV.2010.10.011

11. Bronchiectasis due to ciliary aplasia in Turner's syndrome - PubMed. (n.d.). Retrieved January 18, 2022, from https://pubmed.ncbi.nlm.nih.gov/10770124/

12. Joshi, J., Patil, M., \& Maske, M. (2006). Bronchiectasis in Turner's syndrome . Lung India, 23(3), 121. https://doi.org/10.4103/0970-2113.44403

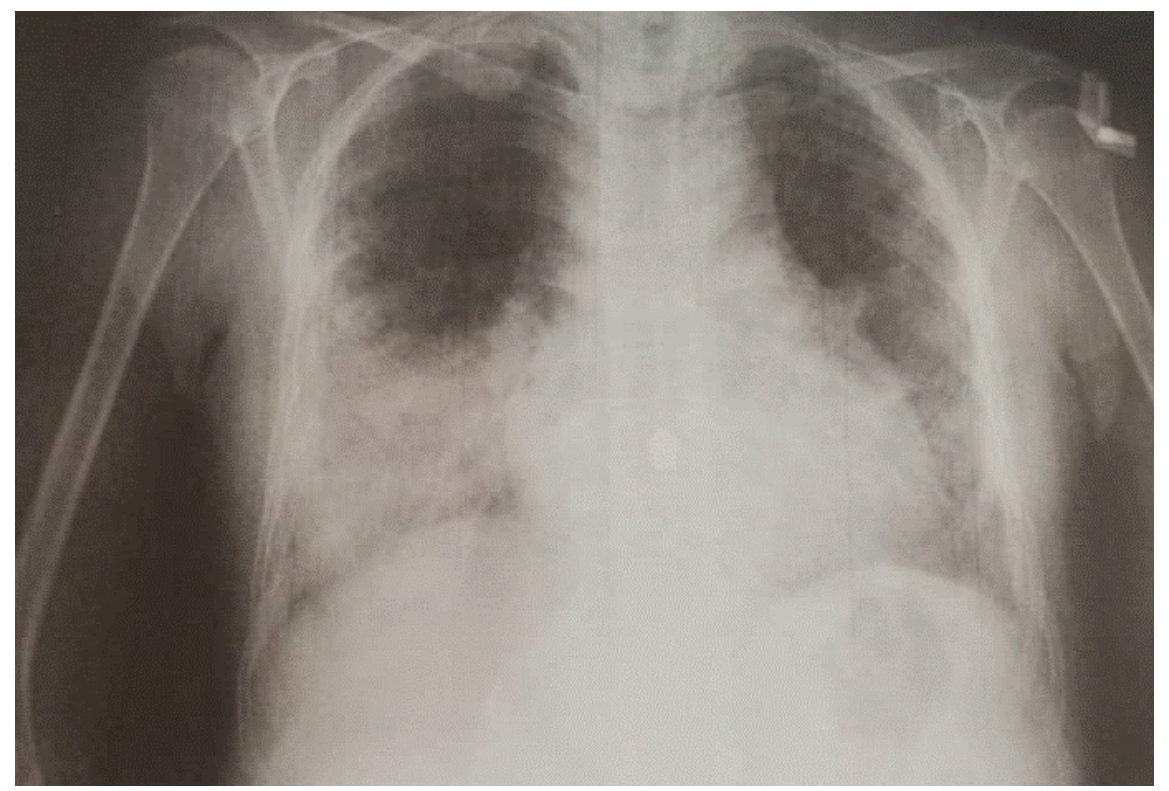




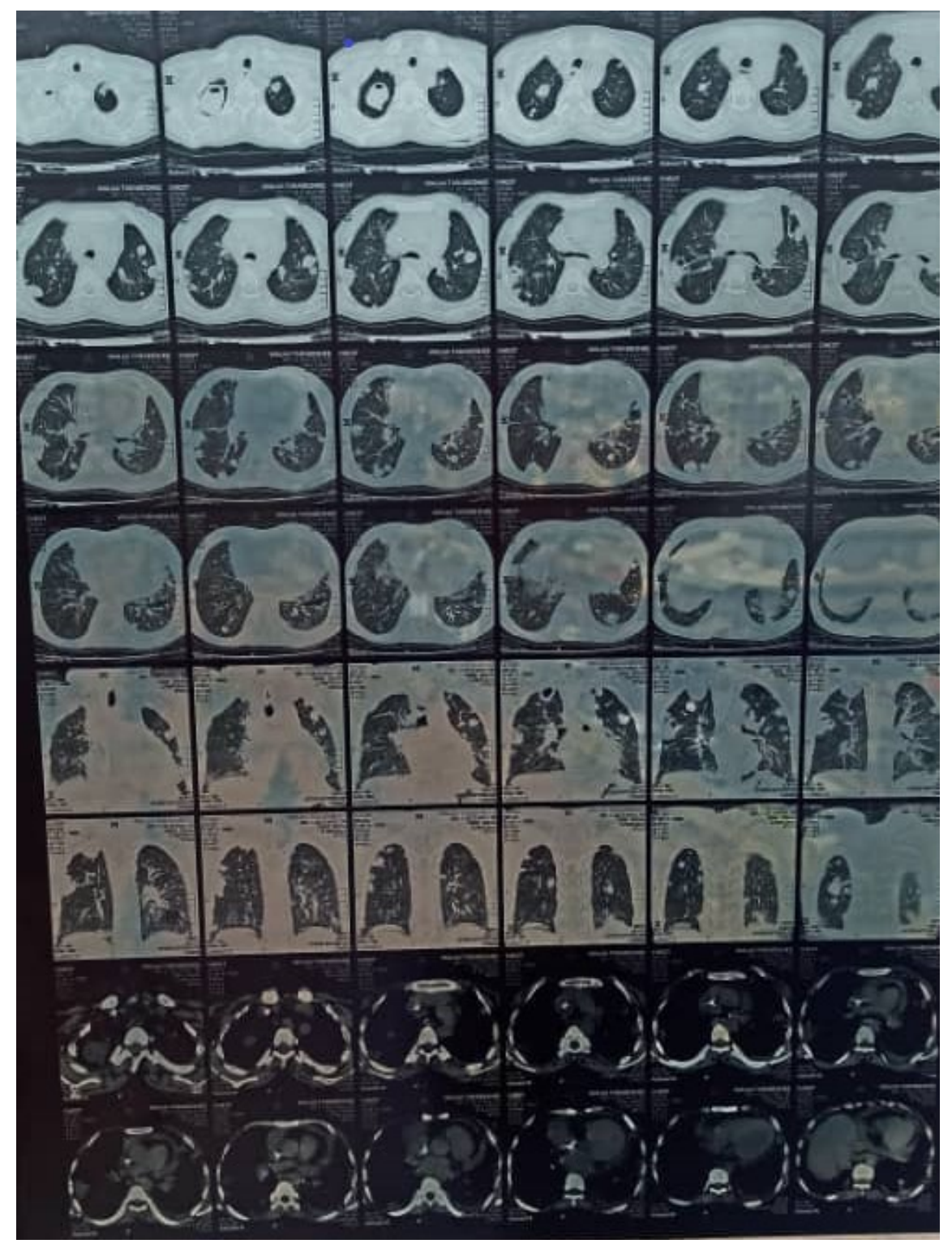

